\title{
Public relations in anaesthesia
}

This misconception can only be corrected by the anaesthetists themselves and no opportunity must be lost to educate our patients and gain their confidence. The first steps must be taken during the pre-operative visit and it is essential that the opportunity to create a favourable impression is not squandered.

Before meeting the patient for the first time a careful scrutiny of the notes will equip the anaesthetist with the vital information needed to instill confidence. If the answers to his questions have previously been supplied by the patient, perhaps on several occasions, unnecessary repetition should be avoided. Not until the relevant information has been assimilated is the anaesthetist ready to meet the patient for the first time.

Dress is important since first impressions are paramount and there is obviously only one opportunity to create one. The anaesthetist who would like to be treated as a Consultant must look like one. A good start is made by addressing the patient by his name and introducing oneself, preferably shaking the patients hand. It is unwise to assume the patient should already know you, however eminent. After a history and examination have been completed the patient will appreciate an explanation, in simple terms, of what to expect before, during and after surgery, including the arrangements for post-operative pain relief.

If the results of pre-operative investigations are favourable this should be stated since it provides reassurance. An explanation of the role that the anaesthetist will play during the operation is reassuring and may come as a surprise. When alternative anaesthetic techniques are available it is important to state clearly which is recommended and why.

If the anaesthetist is unhappy about the condition of the patient and recommends postponement of surgery, a careful explanation to both patient and surgeon is required emphasizing the need to avoid unnecessary risk. The operation may have to be postponed or cancelled due to unforeseen circumstances. This should be communicated to the patient and their relatives without delay so that the anxiety levels do not rise disproportionately. If a surgeon insists on proceeding against the advice of the anaesthetist, claiming that the operation

Roger Eltringham

Consultant Anaesthetist, Gloucestershire Royal Hospital, Great Western Road, Gloucester GLl 3NN, United Kingdom. 
will be life-saving then he should be invited to record this is in the notes and sign it. This is important for the protection of the anaesthetist and sometimes leads to a dramatic change of mind on behalf of the surgeon.

Prior to surgery the anaesthetist should aim to arrive in the theatre well before the scheduled start to allow adequate time to draw up drugs and prepare and test equipment. If the anaesthetist is unavoidably delayed it is wise to call ahead with an explanation and an apology and to give an estimated time of arrival, remembering that a better reception awaits those who exaggerate the possible delay and arrive earlier than expected rather than vice-versa. Failure to observe this simple point of etiquette can result in unnecessary tension which some surgeons will exploit. They should be left in no doubt that, in the given situation, they are extremely fortunate to be operating at all.

As the time of surgery approaches the patient may become nervous and agitated. The creation of a calm atmosphere in the induction room is essential and steps should be taken to avoid interruptions and disturbances at this time. Discussions between members of the surgical team, for example concerning the chances of success, are not unknown as are comments about missing drugs and faulty equipment. These do nothing for the patient's confidence and must be avoided.

By the time general anaesthesia has been established the opportunities for rapport with the patient are reduced, although it is wise to keep in mind that hearing is one of the earliest senses to return if anaesthesia is unduly light. Unnecessary comments about the patient, particular if they are uncomplimentary should, therefore, be strictly avoided as they may be recalled with expensive consequences.

During the course of surgery clear lines of communication with the surgeon should be maintained. It is much better psychology if the anaesthetist draws the surgeon's attention to any deterioration in the patients condition before the surgeon brings this to the attention of the anaesthetist. It is worth remembering that the whispered comments to the anaesthetic nurse are of much greater interest to the surgeon than are the same comments in a normal voice.

The anaesthetist's contribution to intra-operative conversation is a matter requiring judgement and experience and may require adjustment according to the progress of the operation. The surgeon may variously require mood elevation, encouragement, consolation or congratulation according to circumstances and the experienced anaesthetist can play an important role in providing a conducive atmosphere.

When regional anaesthesia is being used and the patient is conscious, additional challenges may face the anaesthetist. Methods of distracting the impatient surgeon whilst awaiting the onset of analgesia require practice whereas the onset of inadequate anaesthesia during the course of the procedure presents a problem requiring immediate resolution. Methods of resolving these situations are discussed.

Musical accompaniment of an operation is frequently initiated by the surgeon but an inappropriate choice can be tactfully reversed by the anaesthetist once the surgeon is scrubbed and immobilised. Doleful music may slow the surgeons progress and a change of tempo may be necessary to restore momentum. If the patient remains conscious throughout surgery the selection can be of added importance.

After the conclusion of surgery the final opportunity for good public relations rests with the post-operative visit. This is an important part of the anaesthetist's work and must not be neglected. It provides an opportunity to assess the patient's condition, give reassurance, and reinforce positive emotions. At this time any feelings of dissatisfaction on behalf of the patient must be promptly dealt with. Complaints must be listened to carefully and, if an apology is deemed necessary, it should be offered before it is demanded. A sympathetic hearing and a polite explanation may be all that is required to convert feelings of injustice into those of gratitude. It is certainly more effective than attempts to apportion blame to others, to avoid responsibility or to cover up mistakes and may subsequently save a great deal of money in legal fees.

Anaesthetists involved in intensive care have an even greater opportunity than others to re-inforce the image of our specialty in the eyes of their colleagues, patients and their relatives. It is important to anticipate their expectations, as common pitfalls await the unwary.

Every opportunity must be taken to keep relatives abreast of developments and they expect a progress report on the condition of the patient each day. Whenever possible this should be given by the same doctor so that a sense of continuity is maintained and rapport and trust can be established.

Whoever interviews the relatives must be capable of providing an overall assessment of the prognosis rather than someone whose expertise is limited to a single organ system. When several teams are treating the same patient the anaesthetist in charge is well placed to fulfil this role. It is easy for the relatives to be ignored as each team leaves this responsibility to others or alternatively for relatives to be given widely differing prognoses, causing confusion and dismay on their part.

When relatives arrive at an intensive care unit for the first time they should be seen by the consultant as soon as possible and not kept waiting outside in a state 
of anxiety. It is wise to provide some advance warning before they enter the ward as the appearance of a ventilated patient surrounded by complex electronic equipment can be frightening. When the prognosis is unknown there is some merit on the erring on the side of caution to avoid disappointment if recovery is more prolonged than anticipated.

Great tact is required when dealing with visiting consultants whose attitude to ICU staff can vary from excessive interference to the point of invisibility. An ICU consultant must be ready to initiate policy decisions in the face of indecision by colleagues. It is not unknown for visiting consultants to develop procrastination into an art form and clear strategies are discussed to avoid the consequent misuse of ICU facilities. A common problem involves the futile prolongation of supportive therapy in the face of a hopeless prognosis and examples are given of how a sympathetic anaesthetist can prevent unnecessary distress by initiating the withdrawal of treatment.

The diagnosis of brain death and the procurement of organs for harvesting can be the subject of great distress unless handled tactfully. An anaesthetist is often called upon to play the pivotal role in coordinating this exercise and common pitfalls are identified to enable this scenario to be enacted with dignity.

Senior consultants can sometimes be heard bewailing the deteriorating attitudes of today's trainees as if this was an inevitable and irreversible trend. As consultants, we have an unique opportunity to influence these attitudes which largely determine the perception of our specialty in the eyes of our patients, colleagues and the public. A good public image is not acquired by chance and few are naturally talented in this field. However, basic principles of good public relations are easily attainable, cost nothing and can lead to great improvements in the perception of our specialty. 


\section{Les relations publiques et l'anesthésie}

Roger Eltringham MB FRCA

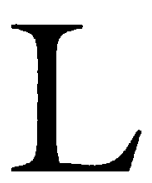

ES progrès constants de l'anesthésie obligent les anesthésistes à développer leurs connaissances théoriques et leur compétence technique. En dépit du fait qu'ils reçoivent, en général, dans les centres universitaires d'enseignement une excellente formation appropriée à l'administration des différentes techniques d'anesthésie, le traitement de la douleur et les soins aux grands malades, les anesthésistes n'accordent pas ou très peu d'attention aux relations publiques. Cette omission est incompréhensible parce que les bienfaits procurés par cet exercice dépassent largement l'effort déployé en terme de succès professionnel, de satisfaction du travail accompli et d'amélioration de l'image de notre discipline.

Comme l'anesthésie a maintenant atteint un très haut profil en débordant dans plusieurs domaines, l'éventualité de conflits avec plusieurs autres spécialités demeure élevée. C'est pour cette raison, qu'il est important pour l'anesthésiste de maintenir de bonnes relations avec le public.

L'observateur attentif peut être témoin des mêmes erreurs répétées mille fois et il est tragique de voir un résident plein de promesses ternir sa réputation sans aucune raison à cause de relations publiques déplorables alors que ceci pourrait être évité s'il prêtait attention à quelques simples principes. Cette communication soulignera les situations délicates et les embûches usuelles et illustrera comment maîtriser les situations difficiles auxquelles nous avons à faire face et même en tirer avantage.

Au cours d'une journée de travail, les anesthésistes agissent en symbiose avec plusieurs groupes dont leurs collègues médecins, le personnel infirmier, la parenté, les adminsitrateurs, les media et, hélas!, avec les avocats. Cependant, ce sont les patients comptent le plus.

Les patients qui se présentent pour une intervention connaissent leur chirurgien et apprécient l'ampleur de ses responsabilités. Toutefois, ils ne connaissent pas forcément leur anesthésiste et n'ont souvent qu'une notion très limitée ou nulle de sa contribution au succès de l'intervention. Certains ne savent même pas que les anesthésistes sont des médecins et pensent même que nous sommes des techniciens travaillant sous les ordres du chirurgien. Ils imaginent que le chirurgien leur permettra d'une certaine façon de passer à travers l'étape de l'anesthésie alors qu'en réalité c'est l'anesthésiste qui les aidera à franchir en toute sécurité l'étape de la chirurgie.

Cette fausse perception ne peut être corrigée que par les anesthésistes eux-mêmes et nous ne devons perdre aucune opportunité pour renseigner nos patients et gagner leur confiance. Ceci débute à la visite préopératoire et il est essentiel de pas gaspiller cette occasion de créer une impression favorable.

Avant de rencontrer son patient pour la première fois, l'étude approfondie du dossier fournira à l'anesthésiste les renseignements nécessaires pour lui inspirer confiance. Si le patient possède déjà les réponses à ses questions, il est parfois inutile de les répéter. L'anesthésiste attendra avant de le rencontrer pour la première fois que le patient ait assimilé tous les renseignements pertinents.

Comme la première impression est décisive et qu'il n'existe qu'un seule opportunité pour la créer, le costume est important. L'anesthésiste qui espère être considéré comme un consultant doit ressembler à un consultant. Pour bien débuter, il s'adressera au patient par son nom et se présentera lui-même, de préférence, en lui serrant sa main. Quelque soit votre notoriété, il serait prétentieux de croire que le patient vous connaît déjà. Une fois l'histoire et l'examen complétés, le patient appréciera une explication, en termes simples, de ce à quoi il doit s'attendre avant, pendant et après la chirurgie en incluant les arrangements qui seront pris pour soulager sa douleur à la période postopératoire.

Si les résultats du bilan paraclinique préopératoire sont favorables, il est bon d'en de l'avertir pour le rassurer. Une explication sur le tâche de l'anesthésiste pendant l'intervention réconfortera et même pourra surprendre le patient. Quand l'anesthésiste peut offrir des techniques anesthésiques de rechange, il est important de mentionner avec clarté lesquelles il recommande et pour quelles raisons.

Si l'anesthésiste n'est pas satisfait de la condition du patient et recommande que l'intervention soit reportée, il soulignera dans son explication destinée aussi bien au patient qu'au chirurgien la nécessité d'écarter tout risque inutile. Des circonstances imprévues peuvent forcer un report ou l'annulation de l'intervention. Elles devraient être communiquées sans délai au patient et à sa famille pour éviter de provoquer une anxiété additonnelle. Si le chirurgien insiste pour 
intervenir malgré l'avis de l'anesthésiste, sous prétexte que l'opération est vitale, il faut l'inviter à l'inscrire au dossier et à signer. Tout en étant importante pour la protection de l'anesthésiste, cette façon de procéder produit parfois un changement dramatique d'attitude de la part du chirurgien.

Avant la chirurgie, l'anesthésiste doit se présenter en salle d'opération bien avant l'heure prévue de l'opération pour la préparation des médicaments et de son équipement et leur vérification. $\mathrm{Si}$, pour des raisons incontrôlables, l'anesthésiste doit retarder, il est sage de téléphoner pour s'expliquer, s'excuser et communiquer le moment de son arrivée; il est bon d'arriver plus tôt que prédit et non vice versa. Le manquement à cette règle d'étiquette peut provoquer une tension inutile que certains chirurgiens ne manqueront pas d'exploiter; il faut leur laisser savoir que dans cette situation particulière, ils sont très chanceux de pouvoir quand même opérer.

A mesure que l'heure de la chirurgie approche, le patient devient parfois nerveux et inquiet. Une atmosphère calme doit régner dans la salle d'induction, et c'est le moment où il faut prendre toutes les mesures pour éviter les interruptions et les dérangements. Il est fréquent d'entendre des discussions sur les chances de succès de l'intervention entre les membres de l'équipe chirurgicale tout comme des commentaires de la part de l'anesthésiste sur le manque d'agents et les défectuosités de l'équipement. Il faut éviter ce type de comportement qui mine la confiance du patient.

L'anesthésie générale de toute évidence réduit les opportunités de communiquer avec le patient, mais est bon de rappeler que l'ouie est le premier des sens à réapparaître lorsque l'anesthésie est trop légère. Le personnel évitera tout commentaire au sujet du patient, particulièrement ceux qui sont peu flatteurs, parce qu'un rappel imprévu pourrait coûter cher.

Pendant la chirurgie, l'anesthésiste doit maintenir une voie de communication intelligible avec le chirurgien. Sur le plan psychologique, il est mieux que ce soit l'anesthésiste qui avertisse le chirurgien que l'état du patient se détériore que le contraire. Il est bon de rappeler ici que les commentaires chuchotés à l'assistant(e) de l'anesthésiste intéressent plus le chirurgien que ceux qui sont prononcés à haute voix.

Pendant l'intervention, la contribution de l'anesthésiste à la conversation est une question de jugement et d'expérience, et doit cadrer avec le déroulement de la chirurgie. Selon les circonstances, le chirurgien a besoin d'être stimulé, encouragé, consolé et félicité, et, à l'occasion, l'attitude de l'anesthésiste expérimenté permettra d'influencer favorablement l'atmosphère de la salle d'opération.
Le patient conscient sous anesthésie régionale présente pour l'anesthésiste des difficultés particulières. Distraire le chirurgien en attendant que l'analgésie soit complète nécessite de l'expérience alors que l'insuffisance de l'analgésie pendant le déroulement de l'intervention exige une attention immédiate. Les moyens de régler ces problèmes sont discutés.

Le chirurgien aime parfois une musique de fond mais son choix peut être modifié avec tact par l'anesthésiste une fois qu'il s'est brossé et immobilisé. Une musique lugubre ralentit le chirurgien et un changement de rythme peut devenir nécessaire pour rétablir sa force d'impulsion. Si le patient demeure conscient pendant l'intervention, le choix de la musique revêt encore plus d'importance.

Une fois la chirurgie complétée, la visite postopératoire fournit la dernière opportunité de maintenir des bonnes relations avec le patient. La visite postopératoire fait partie du rôle de l'anesthésiste et elle ne doit pas être négligée. Elle lui permet d'évaluer l'état du patient, de le réassurer et de renforcer ses impressions positives. C'est le moment de résoudre tout ce qui ressemble à du mécontentement. C'est le moment d'écouter les récriminations, et si des excuses sont justifiées, ils faut les offrir avant que le patient les exige. Un oreille sympathique et une explication courtoise peuvent être tout ce qui est nécessaire pour transformer l'impression d'une injustice en sentiment de gratitude. Cette attitude est certes plus efficace que le rejet du blâme sur les autres, la fuite en face ses responsibilités et l'enterrement de ses erreurs, et, en bout de ligne, elle peut faire épargner beaucoup d'argent.

Les anesthésistes qui travaillent en soins intensifs ont une plus grande opportunité de consolider l'image de notre discipline aux yeux de leurs collègues, des patients et de leur famille. Il important d'anticiper les réactions, car pour ceux qui ne se méfient pas, la voie est parsemée d'embûches.

Comme la famille s'attend à un rapport d'étapes sur la condition du patient, il faut saisir toutes les occasions pour la prévenir de tout nouveau développement. Quand c'est possible, ce rapport devrait toujours être produit par le même médecin de façon à maintenir une continuité qui facilitera l'installation d'un climat de confiance.

Le médecin qui communique avec la famille doit être apte à fournir un point du vue global du pronostic plutôt qu'un médecin dont la compétence se limite à un seul système. Quand plusieurs équipes de spécialistes traitent le même patient, l'anesthésiste qui l'a pris en charge est le mieux placé pour remplir ce rôle. Il est facile d'oublier la famille lorsque chaque membre de l'équipe laisse cette responsabilité aux autres ou 
encore lorsque plusieurs versions du pronostic circulent, ce qui cause de la confusion et du désarroi.

Quand les parents se présentent à l'unité des soins intensifs pour la première fois, ils doivent rencontrer le médecin responsable le plus tôt possible et ne pas être laissés à l'extérieur, ce qui pour effet $\mathrm{d}$,entretenir leur anxiété. Avant de les laisser pénétrer dans l'USI, il est sage de les informer à l'avance parce que la vue d'un patient ventilé et entouré d'appareils électroniques très complexes peut être affolante. Quand le pronostic est douteux, il est préférable d'user de la plus grande réserve pour éviter de désappointer si la récupération se prolongeait.

Il faut user de beaucoup de tact lorsqu'on traite avec des consultant dont l'attitude à l'USI varie entre l'interférence excessive à l'invisibilité la plus totale. En face de l'indécision de ses collègues, l'anesthésisteréanimateur doit être prêt à faire lui-même les gestes qui s'imposent. Certains consultants font de la procrastination une forme d'art; des stratégies précises doivent donc être discutées pour prévenir l'utilisation abusive des soins intensifs. Un traitement de soutien inutile est souvent prolongé même si le pronostic est désespéré; pour protéger de l'angoisse inutile, l'anesthésiste compatissant a souvent donné l'exemple en cessant le traitement.

S'il ne sont pas abordés avec tact, les problèmes qui entourent le diagnostic de mort cérébrale et les dons d'organes peuvent causer beaucoup de chagrin. On demande souvent à l'anesthésiste de servir de coordinateur et la connaissance des embûches les plus courantes permettra de les franchir avec dignité.

Parmi les médecins, les aînés ont critiquent l'attitude des résidents d'aujourd'hui comme si cette tendance était inévitable et irréversible. Nous avons une occasion unique d'influencer les attitudes qui déterminent comment nous sommes perçus par nos patients. Une bonne image aux yeux du public ne s'acquiert pas par hasard et peu nombreux sont ceux qui possèdent un talent naturel. Cependant, les principes fondamentaux des bonnes relations publiques peuvent être acquis facilement, ne coûtent rien et peuvent améliorer nettement la perception de notre spécialité. 